\title{
LAPTM4B polymorphism increases susceptibility to multiple cancers in Chinese populations: a meta-analysis
}

\author{
Cuiju Mo, Yu Lu, Yan Deng, Jian Wang, Li Xie, Taijie Li, Yu He, Qiliu Peng, Xue Qin and Shan Li
}

\begin{abstract}
Background: Lysosome-associated protein transmembrane-4 beta (LAPTM4B) is a novel cancer-related gene. While recent studies have reported that the LAPTMAB polymorphism increased the susceptibility of several cancers, the results remain inconclusive. Therefore, we performed a meta-analysis to systematically summarize the possible association.

Results: The meta-analysis was conducted based on 17 studies in Chinese populations, including 4160 cases and 4148 controls. The relevant studies were searched through electronic databases updated in November 2013. The strength of association between the LAPTM4B polymorphism and susceptibility to multiple cancers was assessed by odds ratio (OR) and $95 \%$ confidence interval $(95 \% \mathrm{Cl})$.

The meta-analysis results suggested that the LAPTMAB polymorphism was significantly associated with overall susceptibility to multiple cancers in all genetic models ( ${ }^{*} 2$ vs. ${ }^{*} 1, \mathrm{OR}=1.53,95 \% \mathrm{Cl}=1.37-1.70 ;{ }^{*} 2 / 2$ vs. ${ }^{*} 1 / 1$, $\mathrm{OR}=2.18,95 \% \mathrm{Cl}=1.72-2.75 ;{ }^{*} 2 / 1 \mathrm{vs} .^{*} 1 / 1, \mathrm{OR}=1.62,95 \% \mathrm{Cl}=1.41-1.86 ;{ }^{*} 2 / 1+{ }^{*} 2 / 2$ vs. ${ }^{*} 1 / 1, \mathrm{OR}=1.70,95 \%$ $\mathrm{Cl}=1.47-1.97 ;{ }^{*} 2 / 2$ vs. $\left.{ }^{*} 2 / 1+{ }^{*} 1 / 1, \mathrm{OR}=1.76,95 \% \mathrm{Cl}=1.50-2.05\right)$. Further subgroup analysis revealed a significant association between the $\angle A P T M 4 B$ polymorphism and cancer susceptibility in the subgroups stratified by control source, cancer type, histopathologic differentiation, and TNM stage.
\end{abstract}

Conclusions: This meta-analysis indicated that the $L A P T M 4 B * 2$ allele was associated with increasing risk of multiple cancers, tumor initiation and development.

Keywords: LAPTM4B, Polymorphism, Cancer, Meta-analysis

\section{Background}

Cancer is one of the leading causes of death in both developed and developing countries, genetic factors contribute significantly to carcinogenesis [1]. Lysosome-associated protein transmembrane- 4 beta $(L A P T M 4 B)$ is a novel cancer-related gene that was cloned from hepatocellular carcinoma (HCC) tissues using fluorescence differential display, rapid amplification of cDNA ends (RACE), and reverse transcription polymerase chain reaction (RTPCR) $[2,3]$. According to BLAST program analysis, the $L A P T M 4 B$ gene is composed of seven exons and six introns mapped to chromosome 8q22 [4]. The full length of messenger RNA for this gene is $2245 \mathrm{bp}$, which encoded a lysosome-associated protein with four

\footnotetext{
* Correspondence: lis8858@126.com

Department of Clinical Laboratory, First Affiliated Hospital of Guangxi Medical University, Nanning, Guangxi 530021, China
}

transmembrane regions. Because it is $46 \%$ homologous with a human lysosome-associated transmembrane- 4 protein (LAPTM4A) at the amino acid level, it was named $L A P T M 4 B$ by the International Committee [5].

In previous studies, $L A P T M 4 B$ mRNA and protein were found to be overexpressed in HCC tissues and expressed at fairly low levels in noncancerous or normal liver tissues. In addition, the expression levels were associated with the differentiation status of HCC; it exhibited higher expression in poor-differentiated HCC than in moderate and well-differentiated HCC [4-6]. Recently, upregulated $L A P T M 4 B$ expression was also found in many other malignant tumors, such as breast cancer [7], pancreatic cancer [8], and gallbladder carcinoma (GBC) [9]. LAP $T M 4 B$ has important roles in many biological processes, including malignant transformation; cell proliferation, invasion, metastasis, and apoptosis; and signal transduction $[9,10]$. 
LAPTM $4 B$ has two alleles: LAPTM $4 B$ *1 (GenBank accession number AY219176) and LAPTM4B *2 (GenBank accession number AY219177) [11]. The difference between allele * 1 and allele *2 is at the 5 'UTR in the first exon; allele "1 contains only a single 19-bp segment, whereas allele "2 has two tandem repeats of the 19-bp segment. LAPTM $4 B$ allele *1 produces a 317-amino acid protein, while allele 2 was predicted to encode a 370 amino acid protein; there are 53 extra amino acids at the N-terminus of the proteins produced by allele *2 [4]. Recent studies have reported that the $L A P T M 4 B$ polymorphism is associated with cancer risk. The $L A P T M 4 B$ "2 allele is reportedly associated with increased risk of such cancers as liver, gastric, colon, and gallbladder cancer, but not with rectal, oesophageal, or pancreatic carcinoma. Given these controversial results and the limits of a single study with a small sample size, we performed the present meta-analysis of all eligible studies in Chinese populations to investigate the association between the $L A P T M 4 B$ polymorphism and susceptibility to multiple cancers.

\section{Methods}

\section{Search strategy}

The relevant studies were searched through the PubMed, Embase, Google Scholar, Wanfang, and China National Knowledge Infrastructure (CNKI) electronic databases updated in November 2013. There were no languages, time period, or sample size restrictions. The search key words were "cancer, carcinoma, tumor, neoplasms", "LAPTM4B, Lysosome-associated protein transmembrane-4", and "polymorphism, mutation, variant". Reference lists of retrieved articles and reviews were checked for other eligible studies. If the same case series was researched by more than one study, only the one with the largest population was included in our study. If one study investigated multiple cancers, each cancer type was counted as a separate comparison in the subgroup stratified by cancer type.

\section{Inclusion and exclusion criteria}

Studies included in the meta-analysis had to meet the following inclusion criteria: (1) original case-control studies; (2) associated the LAPTMAB polymorphism with cancer susceptibility in a Chinese population; (3) provided sufficient information of genotype distribution in the cases and controls; (4) did not repeat reports in the same population. The criteria for exclusion were (1) case reports, reviews, overlapped data, animal or mechanism studies; (2) no genotype frequency or genotype information provided; (3) not enough information for data extraction.

\section{Data extraction}

The following data were independently extracted from all eligible studies by two reviewers (Mo and $\mathrm{Lu}$ ) according to the selection criteria. Disagreements were resolved through group discussion. The data collected from each study were as follows: first author's name, publication year, ethnicity, cancer type, genotyping method, control source, numbers of cases and controls, genotype distribution in cases and controls, and Hardy-Weinberg Equilibrium (HWE). Eligible studies were divided into hospital-based (HB) and population-based (PB) subgroups, according to the control source. Based on the main cancer type of the included studies, cancer types were classified as gastrointestinal cancer (GIC), including oesophageal, gastric, colon, and rectal cancer; gynaecological cancer (GC), including ovarian, cervical, and endometrial carcinoma; liver cancer, lung cancer, breast cancer (BC), and others, including gallbladder carcinoma, pancreatic cancer, nasopharyngeal carcinoma, and lymphoma.

\section{Statistical analysis}

All of the analysis was performed using Stata version 12.0 software (Stata Corp, College Station, TX). The strength of association between the LAPTM $4 B$ polymorphism and susceptibility to multiple cancers was assessed by odds ratio (OR) and $95 \%$ confidence interval $(95 \% \mathrm{CI})$ in all genetic models (allele model: " 2 vs. " 1 ; co-dominant model: " $2 / 2$ vs. " $1 / 1,{ }^{*} 2 / 1$ vs. * $1 / 1$; dominant model: ${ }^{*} 2 / 1+{ }^{*} 2 / 2$ vs. ${ }^{*} 1 / 1$; recessive model: ${ }^{*} 2 / 2$ vs. $\left.{ }^{*} 2 / 1+{ }^{*} 1 / 1\right)$. Subgroup analyses were carried out according to control source, cancer type, tumor grade based on histopathologic differentiation, and tumor stage based on TNM. The stability of the results was assessed using sensitivity analysis by deleting each single study involved in the meta-analysis one at a time to reflect the influence of the individual study to the pooled ORs. Heterogeneity was evaluated by the Chi-square-based Q-statistic and $\mathrm{I}^{2}$ statistic. The DerSimonian-Laird random-effects model was used to assess pooled OR when there was a significant difference in terms of heterogeneity $\left(\mathrm{p}_{\mathrm{Q}}<0.1\right.$ or $\left.\mathrm{I}^{2} \geq 50 \%\right)$. Otherwise, the Mantel-Haenszel fixed-effects model was used. Potential publication bias was estimated by funnel plots and Egger's test. An Egger's test $\mathrm{p}$ value $<0.05$ was considered statistically significant. The HWE was detected by a goodness-of-fit Chi-square test. All $\mathrm{p}$ values were two-sided, and $\mathrm{p}<0.05$ for any test was considered to be statistically significant. This article was prepared based on the PRISMA guidelines, and the checklist was shown in Additional file 1.

\section{Results}

\section{Characteristics of eligible studies}

Figure 1 graphically illustrates the detailed study selection process. A total of 20 published studies associated with the $L A P T M 4 B$ gene polymorphism and susceptibility to multiple cancers were identified for detailed evaluation according to our search criteria. After reading the full articles in detail, three studies were excluded because 


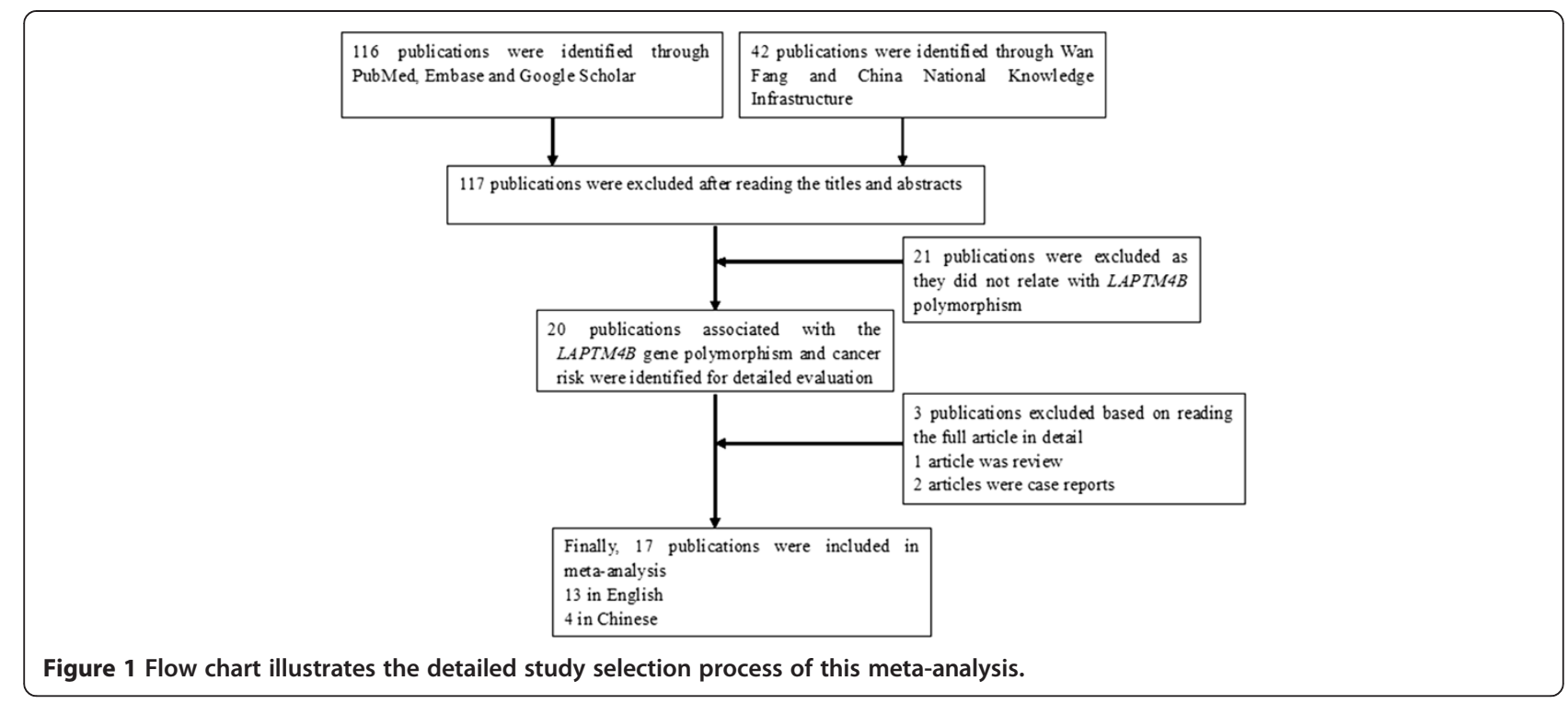

they were case reports [12,13] and a review article [14]. Thus, 17 studies of Chinese populations, totalling 4160 cases and 4148 controls, were included in the metaanalysis [15-31]. Table 1 lists the main characteristics of the included studies. Among these studies, twelve were published in English [17,18,21-29,31] and five were published in Chinese [15,16,19,20,30]. The study of Qi et al. was a postgraduate thesis [16]. The Zhai et al. [17] study only gave the allele frequencies. The study of Cheng et al. [24] included three cancers and shared controls, so the cancer cases were combined into a cancer group in the overall analysis and subgroup analyses stratified by control source and TNM stage, while each cancer was counted as a separate comparison in the subgroup analysis stratified by cancer type. Thirteen of the 17 eligible studies were HB studies and the remaining four were PB studies. The studies focused on the following cancer types: four liver cancer [15-18]; two lung cancer [19,20]; two breast

Table 1 Characteristics of all the eligible studies

\begin{tabular}{|c|c|c|c|c|c|c|c|c|c|c|c|c|}
\hline \multirow{2}{*}{$\begin{array}{l}\text { First } \\
\text { author }\end{array}$} & \multirow[t]{2}{*}{ Year } & \multirow{2}{*}{$\begin{array}{l}\text { Control } \\
\text { source }\end{array}$} & \multirow[t]{2}{*}{ Cancer types } & \multirow{2}{*}{$\begin{array}{l}\text { Genotyping } \\
\text { method }\end{array}$} & \multirow{2}{*}{$\begin{array}{l}\text { Case/ } \\
\text { control }\end{array}$} & \multirow[t]{2}{*}{ HWE } & \multicolumn{3}{|c|}{ Case } & \multicolumn{3}{|c|}{ Control } \\
\hline & & & & & & & ${ }^{*} 1 / 1$ & ${ }^{*} 1 / 2$ & $* 2 / 2$ & ${ }^{*} 1 / 1$ & $* 1 / 2$ & $* 2 / 2$ \\
\hline Sun & 2008 & PB & Liver cancer & PCR & 190/175 & 0.587 & 72 & 110 & 8 & 99 & 67 & 9 \\
\hline Qi & 2010 & $\mathrm{HB}$ & Liver cancer & PCR & $86 / 78$ & 0.799 & 27 & 51 & 8 & 36 & 34 & 7 \\
\hline Zhai & 2012 & PB & Hepatocellular carcinoma & PCR & 102/135 & & 37 & 52 & 13 & & & \\
\hline Wang & 2011 & $\mathrm{HB}$ & Primary liver cancer & PCR & $303 / 515$ & 0.941 & 107 & 156 & 40 & 272 & 205 & 38 \\
\hline Deng & 2005 & $\mathrm{HB}$ & Lung cancer & PCR & $162 / 134$ & 0.285 & 54 & 91 & 21 & 67 & 59 & 8 \\
\hline $\mathrm{Li}$ & 2006 & PB & Lung cancer & PCR & $131 / 104$ & 0.155 & 70 & 56 & 5 & 57 & 36 & 11 \\
\hline $\mathrm{Li}$ & 2012 & $H B$ & Breast cancer & PCR & $208 / 211$ & 0.185 & 90 & 100 & 18 & 129 & 76 & 6 \\
\hline Fan & 2012 & $\mathrm{HB}$ & Breast cancer & PCR & $732 / 649$ & 0.356 & 326 & 342 & 64 & 346 & 262 & 41 \\
\hline Yang & 2012 & PB & Gallbladder carcinoma & PCR & $91 / 155$ & 0.851 & 34 & 45 & 12 & 88 & 57 & 10 \\
\hline Wang & 2013 & $H B$ & Nasopharyngeal carcinoma & PCR & $134 / 327$ & 0.07 & 74 & 48 & 12 & 163 & 145 & 19 \\
\hline Wang & 2010 & $H B$ & Pancreatic cancer & PCR & $58 / 156$ & 0.977 & 24 & 26 & 8 & 74 & 67 & 15 \\
\hline $\mathrm{Xu}$ & 2012 & $\mathrm{HB}$ & Ovarian cancer & PCR & $282 / 365$ & 0.64 & 122 & 115 & 45 & 231 & 108 & 26 \\
\hline Meng & 2011 & $\mathrm{HB}$ & Cervical carcinoma & PCR & $317 / 416$ & 0.835 & 127 & 153 & 37 & 225 & 163 & 28 \\
\hline Meng & 2013 & $H B$ & Endometrial carcinoma & PCR-RFLP & $283 / 378$ & 0.072 & 93 & 135 & 55 & 200 & 140 & 38 \\
\hline Liu & 2007 & $\mathrm{HB}$ & Gastric cancer & PCR & $214 / 350$ & 0.484 & 88 & 107 & 19 & 199 & 133 & 18 \\
\hline Cheng & 2008 & $H B$ & Colon/rectal/esophageal cancer & PCR & $701 / 350$ & 0.484 & 362 & 293 & 46 & 199 & 133 & 18 \\
\hline Sun & 2007 & $\mathrm{HB}$ & Lymphoma & PCR & $166 / 350$ & 0.484 & 72 & 71 & 23 & 199 & 133 & 18 \\
\hline
\end{tabular}


cancer [21,22]; one cervical carcinoma [23]; one colon, rectal, and oesophageal cancers [24]; one gallbladder carcinoma [25]; one gastric cancer [26]; one nasopharyngeal carcinoma [27]; one ovarian cancer [28]; one pancreatic cancer [29]; one lymphoma [30]; and one endometrial carcinoma [31]. All of the patients were confirmed by histopathologic examination. Genotype distributions in the controls of all the studies were in agreement with HWE.

\section{Results of meta-analysis}

As shown in Table 2, obvious heterogeneity existed among the studies; therefore, the random-effects model was used to assess pooled OR. Overall, the $L A P T M 4 B$ polymorphism was significantly associated with overall susceptibility to multiple cancers in the Chinese population in all genetic models ( 2 vs. ${ }^{*} 1, \mathrm{OR}=1.53,95 \% \mathrm{CI}=1.37$ $1.70, \mathrm{p}<0.001$, Figure 2 ; ${ }^{*} 2 / 2$ vs. ${ }^{*} 1 / 1, \mathrm{OR}=2.18,95 \%$ $\mathrm{CI}=1.72-2.75, \mathrm{p}<0.001 ;{ }^{*} 2 / 1$ vs. ${ }^{*} 1 / 1, \mathrm{OR}=1.62,95 \%$ $\mathrm{CI}=1.41-1.86, \mathrm{p}<0.001 ; " 2 / 1+* 2 / 2$ vs. ${ }^{*} 1 / 1, \mathrm{OR}=1.70$, $95 \% \mathrm{CI}=1.47-1.97, \mathrm{p}<0.001 ; \quad * 2 / 2$ vs. ${ }^{*} 2 / 1+{ }^{*} 1 / 1$, $\mathrm{OR}=1.76,95 \% \mathrm{CI}=1.50-2.05, \mathrm{p}<0.001)$.

The subgroup analysis results are provided in Table 3. In the subgroup analysis by control source, there was a significant association between the LAPTM4B polymorphism and susceptibility to multiple cancers in both the HB (all genetic models) and PB (allele, heterozygote, and dominant comparison models) subgroups. When stratified by cancer type, increased cancer susceptibility was found in all genetic models for liver cancer and GC. There was a significant association with risk of breast cancer in four genetic comparisons, except the homozygous model (" $2 / 2$ vs. ${ }^{*} 1 / 1, \mathrm{OR}=2.41,95 \% \mathrm{CI}=0.97-6.01$, $\mathrm{p}=0.059)$. In the lung cancer and GIC subgroups, significant associations were only found in the heterozygote model (lung cancer: ${ }^{*} 2 / 1$ vs. ${ }^{*} 1 / 1, \mathrm{OR}=1.56,95 \% \mathrm{CI}=$ 1.11-2.29, $\mathrm{p}=0.012$; GIC: * $2 / 1$ vs. $" 1 / 1, \mathrm{OR}=1.33,95 \%$ $\mathrm{CI}=1.03-1.73, \mathrm{p}=0.030$ ). Moreover, to investigate the relationship between $L A P T M 4 B$ genotypes and cancer clinical parameters, we analysed the distribution of histopathologic differentiation and classification of TNM and found that the LAPTM4B polymorphism was associated with histopathologic differentiation (heterozygote and dominant models). Significant association was found in the allele, heterozygote, and dominant comparison models in the TNM I-II subgroup and in all genetic models in the TNM III-IV subgroup.

\section{Sensitivity analysis}

In order to test whether the modification of the inclusion criteria of the meta-analysis affected the pooled ORs, we performed a sensitivity analysis by deleting each single study involved in the meta-analysis, one at a time. The pooled ORs were not materially altered, suggesting that our results were statistically robust (data not shown).

\section{Publication bias}

Egger's test and Begg's funnel plot were conducted to estimate the possible publication bias of the studies. The Egger's test results suggested no publication bias for all the genetic models (Table 2). Figure 3 shows the shape of the Begg's funnel plots for the allele comparison " 2 vs. " 1 of the $L A P T M 4 B$ polymorphism in the overall population, indicating no obvious publication bias in the meta-analysis.

\section{Discussion}

Meta-analysis is a valuable tool for estimating the effect of a genetic factor on the risk of a disease when the individual sample sizes are small $[32,33]$. While previous studies have reported that $L A P T M 4 B$ polymorphism is associated with the risk of several cancers, the results are controversial. Therefore, we carried out this metaanalysis of 17 studies involving 8308 samples, identified through a database search. Overall, we found a significant association between the $L A P T M 4 B$ polymorphism and susceptibility to multiple cancers in all genetic models. This support from our meta-analysis indicates that the $L A P T M 4 B$ polymorphism is a possible susceptibility factor for multiple cancers in the Chinese population, especially for liver cancer and GC.

Cancer is a multifactorial disease and its etiology is still unclear [1]. LAPTM4B is a novel cancer-associated gene belonging to the mammalian LAPTM family and upregulated in a wide variety of solid tumors $[11,34]$. LAPTM $4 B$ has been shown to promote the transition from $\mathrm{G} 1$ to $\mathrm{S}$ phase and cell malignant proliferation in LAPTM4B cDNA transfected cells by upregulating the

Table 2 Meta-analysis of eligible studies included in the study

\begin{tabular}{|c|c|c|c|c|c|c|c|c|c|}
\hline \multirow[t]{2}{*}{ Comparison models } & \multirow[t]{2}{*}{ No. of studies } & \multicolumn{3}{|c|}{ Test of association } & \multirow[t]{2}{*}{ Mode } & \multicolumn{3}{|c|}{ Test of heterogeneity } & \multirow{2}{*}{$\begin{array}{l}\text { Publication bias } \\
\text { P }_{\text {bias }}\end{array}$} \\
\hline & & OR & $95 \% \mathrm{Cl}$ & $P$ value & & $x^{2}$ & $\mathrm{P}_{\mathrm{Q}}$ Value & $\mathrm{I}^{2}$ & \\
\hline${ }^{*} 2$ vs. ${ }^{*} 1$ & 17 & 1.53 & $1.37-1.70$ & $<0.001$ & $\mathrm{R}$ & 40.26 & 0.001 & 60.3 & 0.705 \\
\hline${ }^{*} 2 / 2$ vs. ${ }^{*} 1 / 1$ & 16 & 2.18 & $1.72-2.75$ & $<0.001$ & $\mathrm{R}$ & 27.30 & 0.026 & 45.0 & 0.360 \\
\hline${ }^{*} 2 / 1$ vs. ${ }^{*} 1 / 1$ & 16 & 1.62 & $1.41-1.86$ & $<0.001$ & $\mathrm{R}$ & 31.27 & 0.008 & 52.0 & 0.595 \\
\hline${ }^{*} 2 / 1+{ }^{*} 2 / 2$ vs. ${ }^{*} 1 / 1$ & 16 & 1.70 & $1.47-1.97$ & $<0.001$ & $\mathrm{R}$ & 38.05 & 0.001 & 60.6 & 0.698 \\
\hline${ }^{*} 2 / 2$ vs. ${ }^{*} 2 / 1+{ }^{*} 1 / 1$ & 16 & 1.76 & $1.50-2.05$ & $<0.001$ & $\mathrm{R}$ & 22.05 & 0.110 & 32.0 & 0.265 \\
\hline
\end{tabular}

OR odds ratio, 95\% Cl 95\% confidence interval, $R$ random-effects model. 


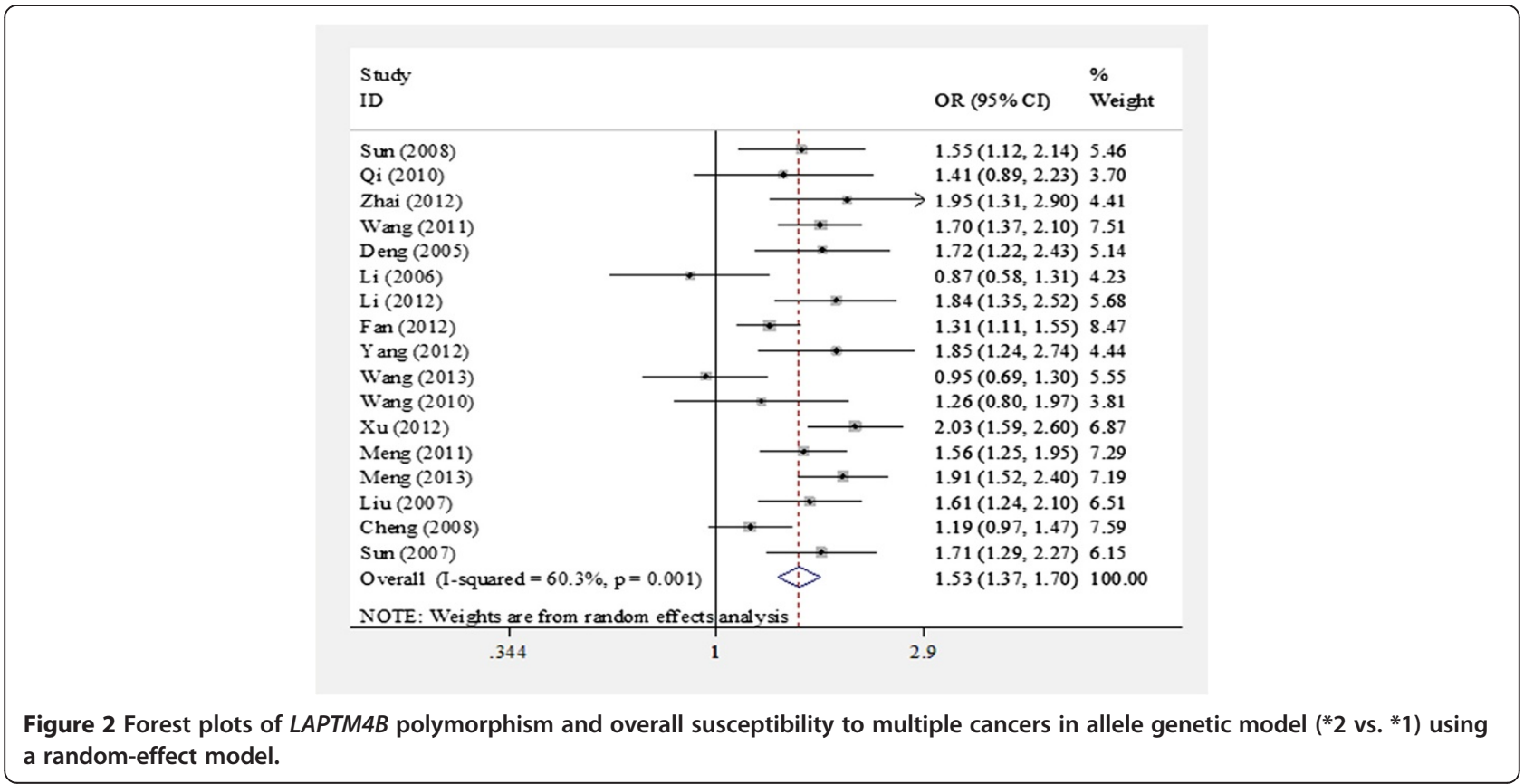

expression of cyclin D1 and E [35]. In addition, the overexpression of $L A P T M 4 B$ may activate the integrin- $\alpha 6$ mediated PI3K-AKT signalling pathway and upregulate some proto-oncogenes such as c-myc, c-jun, and c-fos, which regulate apoptosis and cell proliferation [36]. Moreover, knockdown $L A P T M 4 B$ and mutated PPRP motif in the $\mathrm{N}$-terminal region of $L A P T M 4 B$ could attenuate its role in tumorigenesis and metastasis [37]. Previous studies have revealed that the different 19-bp sequence between the " 2 and " 1 alleles might act as a cis-element, involved in genetic transcriptional regulation. In addition, the extra 53 amino acids at the protein $\mathrm{N}$-terminus produced by LAPTM $4 B^{*} 2$ could influence the biological activity and function of tumor cells and then induce oncogenic susceptibility [5].

Previous studies have reported that the increased risk for individuals carrying allele "2 were significantly associated with a susceptibility to liver cancer [17-20], breast cancer [23,24], cervical carcinoma [25], gallbladder carcinoma [27], gastric cancer [28], ovarian cancer [29], lymphoma [32], and endometrial carcinoma [33]. However, no statistical differences were found in nasopharyngeal carcinoma [29] or pancreatic cancer [31]. Deng [21] found that $L A P T M 4 B^{*} 2$ was a risk factor for lung cancer, while Li [22] reported that LAPTM4B*2 was not closely associated with a susceptibility to lung cancer. Furthermore, the Cheng study indicated that the LAPTM $4 B$ polymorphism was associated with the development of colon cancer, but not with rectal or oesophageal cancers [26]. Considering these controversial results, we performed the present meta-analysis to establish a more conclusive association between the $L A P T M 4 B$ polymorphism and cancer susceptibility.

To the best of our knowledge, this meta-analysis is the first study to investigate the association between the $L A P T M 4 B$ polymorphism and susceptibility to multiple cancers in a large number of Chinese populations. Our results suggested that the $L A P T M 4 B^{*} 2$ allele was associated with a risk of susceptibility to multiple cancers, which is consistent with the most of the original research. Significant heterogeneity was observed in all of the genetic model comparisons, which might be due to the control sources and mix of cancers. Most of the studies used hospital-based patients as controls, who were not strictly healthy individuals and could not represent the general population. In this meta-analysis, many cancers were only researched once, and some polymorphisms may play different roles in different cancers. After subgroup analyses were conducted to consider the evidence of heterogeneity, some heterogeneity in the control source and cancer type subgroups was found. Therefore, we speculated that environment components and difference of clinicopathological characteristic might contribute to the heterogeneity. In order to assess the influence of the individual data set to the pooled ORs, a sensitivity analysis was carried out by deleting each single study involved in the meta-analysis each time. The results did not alter, suggesting that our meta-analysis results were stable and credible.

In further subgroup analyses, we found that the $L A P T M 4 B$ polymorphism was associated with $\mathrm{HB}$ or PB study design, liver cancer, lung cancer, breast cancer, 
Table 3 Stratified analysis of LAPTM4B gene polymorphism on cancer susceptibility

\begin{tabular}{|c|c|c|c|c|c|c|c|c|c|c|c|c|c|c|c|c|}
\hline & \multirow[t]{2}{*}{$\mathrm{N}$} & \multicolumn{3}{|c|}{${ }^{*} 2$ vs. ${ }^{*} 1$} & \multicolumn{3}{|c|}{${ }^{*} 2 / 2$ vs. ${ }^{*} 1 / 1$} & \multicolumn{3}{|c|}{${ }^{*} 2 / 1$ vs. ${ }^{*} 1 / 1$} & \multicolumn{3}{|c|}{${ }^{*} 2 / 1+{ }^{*} 2 / 2$ vs. ${ }^{*} 1 / 1$} & \multicolumn{3}{|c|}{${ }^{*} 2 / 2$ vs. $* 2 / 1+* 1 / 1$} \\
\hline & & OR $(95 \% \mathrm{Cl})$ & $\mathrm{p}$ & $\mathrm{P}_{\mathrm{Q}}$ & OR $(95 \% \mathrm{Cl})$ & $\mathrm{p}$ & $\mathrm{P}_{\mathrm{Q}}$ & OR $(95 \% \mathrm{Cl})$ & $\mathrm{p}$ & $\mathrm{P}_{\mathrm{Q}}$ & OR $(95 \% \mathrm{Cl})$ & $\mathbf{p}$ & $\mathrm{P}_{\mathrm{Q}}$ & OR $(95 \% \mathrm{Cl})$ & $\mathbf{p}$ & $\mathrm{P}_{\mathrm{Q}}$ \\
\hline \multicolumn{17}{|l|}{ Control source } \\
\hline $\mathrm{HB}$ & 13 & $1.53(1.36-1.73)$ & $<0.001$ & 0.002 & $2.33(1.96-2.76)$ & $<0.001$ & 0.234 & $1.58(1.36-1.84)$ & $<0.001$ & 0.007 & $1.67(1.52-1.83)$ & $<0.001$ & 0.001 & $1.86(1.58-2.19)$ & $<0.001$ & 0.612 \\
\hline PB & 4 & $1.49(1.06-2.092)$ & 0.020 & 0.023 & $1.16(0.35-3.80)$ & 0.811 & 0.016 & $1.87(1.40-2.49)$ & $<0.001$ & 0.247 & $1.72(1.09-2.72)$ & 0.019 & 0.073 & $0.88(0.30-2.54)$ & 0.806 & 0.029 \\
\hline \multicolumn{17}{|l|}{ Cancer type } \\
\hline Liver cancer & 4 & $1.66(1.43-1.94)$ & $<0.001$ & 0.714 & $2.15(1.42-3.25)$ & $<0.001$ & 0.312 & $2.03(1.61-2.57)$ & $<0.001$ & 0.847 & $2.06(1.64-2.58)$ & $<0.001$ & 0.963 & $1.52(1.02-2.24)$ & 0.037 & 0.220 \\
\hline Lung cancer & 2 & $1.24(0.63-2.41)$ & 0.534 & 0.013 & $1.13(0.13-9.50)$ & 0.912 & 0.003 & $1.59(1.11-2.29)$ & 0.012 & 0.268 & $1.49(0.77-2.89)$ & 0.234 & 0.058 & $0.90(0.14-5.90)$ & 0.915 & 0.007 \\
\hline Breast cancer & 2 & $1.51(1.09-2.11)$ & 0.014 & 0.058 & $2.41(0.97-6.01)$ & 0.059 & 0.075 & $1.49(1.23-1.81)$ & $<0.001$ & 0.187 & $1.55(1.29-1.87)$ & $<0.001$ & 0.101 & $1.64(1.13-2.38)$ & 0.009 & 0.116 \\
\hline GC & 3 & $1.81(1.58-2.07)$ & $<0.001$ & 0.253 & $2.90(2.16-3.90)$ & $<0.001$ & 0.638 & $1.89(1.56-2.29)$ & $<0.001$ & 0.584 & $2.07(1.73-2.48)$ & 0.007 & 0.400 & $2.14(1.62-2.84)$ & $<0.001$ & 0.716 \\
\hline $\mathrm{GIC}$ & 4 & $1.26(0.97-1.65)$ & 0.084 & 0.008 & $1.49(0.77-2.88)$ & 0.236 & 0.024 & $1.33(1.03-1.73)$ & 0.030 & 0.084 & $1.36(1.00-1.84)$ & 0.050 & 0.020 & $1.32(0.76-2.30)$ & 0.331 & 0.069 \\
\hline Others & 4 & $1.39(1.01-1.92)$ & 0.043 & 0.020 & $2.32(1.56-3.46)$ & $<0.001$ & 0.257 & $1.26(0.81-1.96)$ & 0.316 & 0.020 & $1.39(0.89-2.19)$ & 0.150 & 0.011 & $2.10(1.43-3.09)$ & $<0.001$ & 0.546 \\
\hline \multicolumn{17}{|c|}{ Histopathologic differentiation } \\
\hline poor & 4 & $1.61(0.96-2.70)$ & 0.069 & 0.006 & $2.34(0.64-8.59)$ & 0.200 & 0.008 & $2.03(1.10-3.75)$ & 0.024 & 0.050 & $2.07(1.05-4.12)$ & 0.037 & 0.015 & $1.56(0.62-3.95)$ & 0.346 & 0.060 \\
\hline moderate & 4 & $1.29(0.96-1.72)$ & 0.091 & 0.341 & $0.98(0.44-2.20)$ & 0.959 & 0.333 & $1.83(1.23-2.74)$ & 0.003 & 0.527 & $1.67(1.13-2.47)$ & 0.010 & 0.341 & $0.73(0.33-1.59)$ & 0.425 & 0.523 \\
\hline well & 4 & $1.48(0.88-2.50)$ & 0.143 & 0.060 & $2.14(0.53-8.60)$ & 0.284 & 0.045 & $1.60(1.00-2.55)$ & 0.049 & 0.172 & $1.60(1.03-2.50)$ & 0.037 & 0.121 & $1.35(0.67-2.73)$ & 0.398 & 0.146 \\
\hline \multicolumn{17}{|l|}{ TNM stage } \\
\hline$|-| \mid$ & 8 & $1.38(1.04-1.84)$ & 0.028 & 0.006 & $1.89(0.89-4.00)$ & 0.097 & 0.005 & $1.43(1.16-1.77)$ & 0.001 & 0.415 & $1.49(1.22-1.82)$ & $<0.001$ & 0.096 & $1.61(0.81-3.18)$ & 0.175 & 0.008 \\
\hline III-IV & 8 & $1.56(1.31-1.87)$ & $<0.001$ & 0.032 & $2.23(1.67-2.98)$ & $<0.001$ & 0.075 & $1.79(1.40-2.28)$ & $<0.001$ & 0.047 & $1.86(1.44-2.40)$ & $<0.001$ & 0.017 & $1.70(1.29-2.23)$ & $<0.001$ & 0.276 \\
\hline
\end{tabular}

OR odds ratio, $95 \%$ Cl 95\% confidence interval, $P_{Q} \mathrm{p}$ value for heterogeneity, HB hospital-based, PB population-based, GC Gynecological cancer, GIC Gastrointestinal cancer, TNM Tumor, Node, Metastasis. 


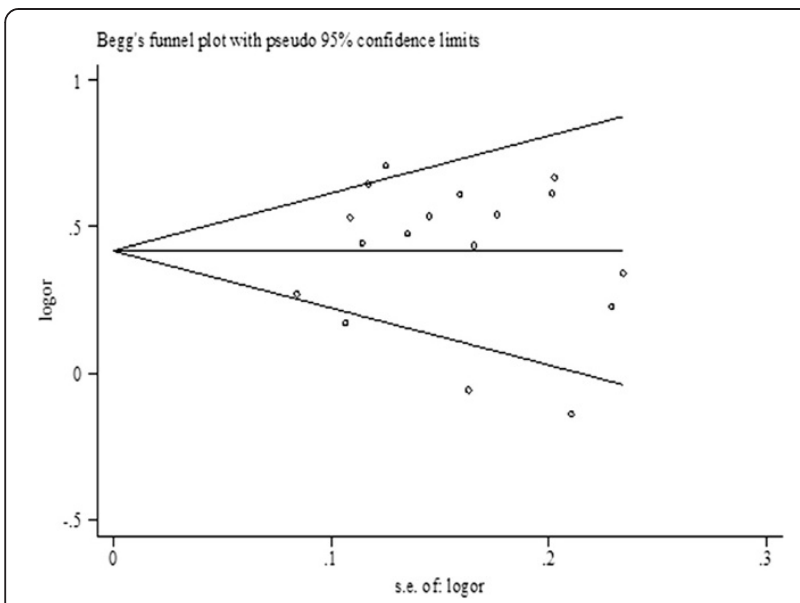

Figure 3 Funnel plot to assess the publication bias of the meta-analysis in allele genetic model $\left({ }^{*} 2\right.$ vs. $\left.{ }^{*} 1\right)$.

GC, and GIC. Two studies researched the relationship of the LAPTM $4 B$ polymorphism with lung and breast cancers, respectively, and they may lack power of statistical analysis. Thirteen studies researched the relationship of LAPTM $4 B$ polymorphism with cancer histopathologic differentiation, of which four provided gene frequency data. Eight studies researched the association between $L A P T M 4 B$ polymorphism and tumor TNM stage, and only three found that $L A P T M 4 B$ polymorphism was significantly associated with cancer histopathologic differentiation and TNM stage, while the present meta-analysis indicated that $L A P T M 4 B$ polymorphism was associated with histopathologic differentiation and TNM stage. The small sample sizes in the individual original research studies might account for the false positive correlation. Thus, further studies with larger sample sizes and better design are needed.

Our meta-analysis has several advantages. First, this is the first systematic review of the association between $L A P T M 4 B$ polymorphism and susceptibility to multiple cancers, and it is statistically more powerful than any single study. Second, all of the eligible studies researched Chinese populations. The participants have the same genetic background, which can reduce the effects of ethnicity on pooled ORs. Third, the distributions of the genotypes in the controls of the included studies met HWE, which increased the statistical power of the meta-analysis. Lastly, we analysed subgroups stratified by control source, cancer types, histopathologic differentiation, and TNM stage. Furthermore, the sensitivity analysis and publication bias analysis indicated that our meta-analysis results were stable and credible. However, there were still some limitations. First, the meta-analysis was based on unadjusted data; the controls were not strictly matched with the cases in some of the original research, which might have caused some bias. Second, there is notable heterogeneity among studies in the overall analysis and in some subgroup analysis, which could lead to some skewed results. Third, the sample size of some of the subgroups was relatively small. In addition, only one study investigated the association in several human cancers. Given these limitations, the present meta-analysis results should be considered with caution. Further studies with larger sample sizes, more cancer types, and other populations are needed to replicate our results.

\section{Conclusion}

This meta-analysis indicates that the $L A P T M 4 B^{*} 2$ allele is associated with elevated susceptibility to multiple cancers. In addition, our results indicate a significant association between the LAPTM4B polymorphism and cancer susceptibility in the subgroups stratified by control source, cancer type, tumor stage based on TNM, and tumor histopathologic differentiation. All of these results indicate that $L A P T M 4 B$ may be an important biomarker for tumor initiation and development.

\section{Consent}

Written informed consent was obtained from the patient for the publication of this report and any accompanying images.

\section{Additional file}

\section{Additional file 1: PRISMA Checklist.}

\section{Abbreviations}

LAPTM4B: Lysosome-associated protein transmembrane-4 beta; HWE: Hardy-Weinberg Equilibrium; HB: Hospital-based; PB: Population-based; GIC: Gastrointestinal cancer; GC: Gynaecological cancer; BC: Breast cancer.

Competing interests

All authors declare that they have no competing interests.

\section{Authors' contributions}

$C M, Y L$, and $X Q$ conceived and designed the experiments; $S L, Y D$, and JW performed the experiments; $\mathrm{LX}, \mathrm{TL}$, and $\mathrm{XQ}$ analyzed the data; $\mathrm{YH}, \mathrm{QP}$, and JW contributed reagents/materials/analysis tools; and CM and SL wrote the manuscript. All of the authors read and approved the final manuscript.

\section{Acknowledgment}

This research was supported by the National Natural Science Foundation of China (No. 81260302).

Received: 5 January 2014 Accepted: 10 April 2014 Published: 18 April 2014

\section{References}

1. Bredberg A: Cancer: more of polygenic disease and less of multiple mutations? A quantitative viewpoint. Cancer 2011, 117(3):440-445.

2. Zhang J, Liu J, Han Y, Zhang N, Rui J, Jin C, Zhou R: Screening of novel hepatocellular carcinomaassociated genes. J Beijing Med Univ 2001, 33(1):54-57.

3. Liu JJ, Zhang J, Zhang N: Identification of new hepatocellular carcinoma related genes by fluorescent differential display. J Beijing Med Univ 2000, 32(5):411-414 
4. Shao GZ, Zhou RL, Zhang QY, Zhang Y, Liu JJ, Rui JA, Wei X, Ye DX: Molecular cloning and characterization of LAPTM4B, a novel gene upregulated in hepatocellular carcinoma. Oncogene 2003, 22(32):5060-5069.

5. Liu J, Zhou R, Zhang N, Rui J, Jin C: Biological function of a novel gene overexpressed in human hepatocellular carcinoma. Chin Med J (Engl) 2000, 113(10):881-885.

6. Peng C, Zhou RL, Shao GZ, Rui JA, Wang SB, Lin M, Zhang S, Gao ZF: Expression of lysosome-associated protein transmembrane 4B-35 in cancer and its correlation with the differentiation status of hepatocellular carcinoma. World J Gastroenterol 2005, 11(18):2704-2708.

7. Li Y, Zou L, Li Q, Haibe-Kains B, Tian R, Desmedt C, Sotiriou C, Szallasi Z, Iglehart JD, Richardson AL, Wang ZC: Amplification of LAPTM4B and YWHAZ contributes to chemotherapy resistance and recurrence of breast cancer. Nat Med 2010, 16(2):214-218.

8. Zhang G, Liang Y, Huang Y, Chen Y, Zhou R: Elevated lysosome-associated protein transmembrane-4beta-35 is an independent prognostic marker in pancreatic carcinoma. J Int Med Res 2012, 40(4):1275-1283.

9. Zhou L, He XD, Yu JC, Zhou RL, Yang H, Qu Q, Rui JA: Overexpression of LAPTM4B promotes growth of gallbladder carcinoma cells in vitro. Am $J$ Surg 2010, 199(4):515-521.

10. Zhou L, He XD, Yu JC, Zhou RL, Xiong FX, Qu Q, Rui JA: Expression of LAPTM4B in gallbladder carcinoma cells: the role in invasive potential. Hepatogastroenterology 2010, 57(98):207-211

11. Liu X, Zhou R, Zhang Q, Zhang Y, Shao G, Jin Y, Zhang S, Lin M, Rui J, Ye D: [Identification and characterization of LAPTM4B encoded by a human hepatocellular carcinoma-associated novel gene]. Beijing Da Xue Xue Bao 2003, 35(4):340-347.

12. Yang $H$, Zhai $G$, Ji X, Xiong F, Su J, McNutt MA: LAPTM4B allele *2 is a marker of poor prognosis following hepatic tumor resection for hepatocellular carcinoma. PLoS One 2012, 7(4):e34984.

13. Zhai G, Yan K, Ji X, Xu W, Yang J, Xiong F, Su J, McNutt MA, Yang H: LAPTM4B allele *2 is a marker of poor prognosis for gallbladder carcinoma. PLoS One 2012, 7(9):e45290.

14. Yang $Y, G$ G: Association of LAPTM4B gene polymorphism with human tumors. Pract Oncol 2013, 27(2):172-176.

15. Sun G, Li Z, Hao W, Niu J, Yin J, Yan Y: Relationship between lysosomeassociated protein transmembrane $4 \beta$ polymorphism and Susceptmmty to liver cancer. World Chin J Digestology 2008, 16(8):908-911.

16. Qi R, Shi X: LAPTM4B gene polymorphism and liver cancer susceptibility (postgraduate's thesis). 2010

17. Zhai G, Yang H, Ji X, Xiong F, Su J, McNutt MA, Li X: Correlation of LAPTM4B polymorphisms with hepatocellular carcinoma in Chinese patients. Med Oncol 2012, 29(4):2744-2749.

18. Wang S, Zhang QY, Zhou RL: Relationship between LAPTM4B gene polymorphism and susceptibility of primary liver cancer. Ann Oncol 2012, 23(7):1864-1869.

19. Deng LJ, Zhang QY, Liu B, Zhou RL: [Relationship between LAPTM4B gene polymorphism and susceptibility of lung cancer]. Beijing Da Xue Xue Bao 2005, 37(3):302-305

20. Li C, Zhou Q, Wang Y, Chen X, Yang X, Zhu D: [Relationship between LAPTM4B gene polymorphism and susceptibility of lung cancer]. Zhongguo Fei Ai Za Zhi 2006, 9(2):109-112.

21. Fan M, Liu Y, Zhou R, Zhang Q: Association of LAPTM4B gene polymorphism with breast cancer susceptibility. Cancer Epidemiol 2012, 36(4):364-368.

22. Li X, Kong $X$, Chen $X$, Zhang N, Jiang L, Ma T, Yang Q: LAPTM4B allele *2 is associated with breast cancer susceptibility and prognosis. PLOS One 2012, 7(9):e44916.

23. Meng F, Song H, Luo C, Yin M, Xu Y, Liu H, Zhou R, Lou G: Correlation of LAPTM4B polymorphisms with cervical carcinoma. Cancer 2011, 117(12):2652-2658

24. Cheng XJ, Xu W, Zhang QY, Zhou RL: Relationship between LAPTM4B gene polymorphism and susceptibility of colorectal and esophageal cancers. Ann Oncol 2008, 19(3):527-532.

25. Yang $H$, Zhai G, Ji X, Xiong F, Su J, McNutt MA: Correlation of LAPTM4B polymorphisms with gallbladder carcinoma susceptibility in Chinese patients. Med Oncol 2012, 29(4):2809-2813.

26. Liu Y, Zhang QY, Qian N, Zhou RL: Relationship between LAPTM4B gene polymorphism and susceptibility of gastric cancer. Ann Oncol 2007, 18(2):311-316
27. Wang B, Xu J, Zhou R, Zhang Q: Association of LAPTM4B gene polymorphism with nasopharyngeal carcinoma susceptibility in a Chinese population. Med Oncol 2013, 30(1):470.

28. Xu Y, Liu Y, Zhou R, Meng F, Gao Y, Yang S, Li X, Yang M, Lou G: LAPTM4B polymorphisms is associated with ovarian cancer susceptibility and its prognosis. Jpn J Clin Oncol 2012, 42(5):413-419.

29. Wang S, Zhang Q: Association of lysosome associated protein transmembrane 4 beta gene polymorphism with the risk of pancreatic cancer. Chin J Cancer Res 2010, 22(4):291-295.

30. Sun L, Zhang Q, Liu Y, Qian N: Relationship between Human Novel Gene LAPTM4B gene polymorphism and susceptibility of Lymphoma. Cancer Res Prev Treat 2007, 34(4):245-248.

31. Meng F, Li H, Zhou R, Luo C, Hu Y, Lou G: LAPTM4B gene polymorphism and endometrial carcinoma risk and prognosis. Biomarkers 2013, 18(2):136-143.

32. Lohmueller KE, Pearce CL, Pike M, Lander ES, Hirschhorn JN: Meta-analysis of genetic association studies supports a contribution of common variants to susceptibility to common disease. Nat Genet 2003, 33(2):177-182.

33. Parkin DM, Bray F, Ferlay J, Pisani P: Global cancer statistics, 2002. CA Cancer J Clin 2005, 55(2):74-108.

34. Kasper G, Vogel A, Klaman I, Grone J, Petersen I, Weber B, Castanos-Velez E, Staub E, Mennerich D: The human LAPTM4b transcript is upregulated in various types of solid tumours and seems to play a dual functional role during tumour progression. Cancer Lett 2005, 224(1):93-103.

35. He J, Shao G, Zhou R: [Effects of the novel gene, LAPTM4B, highly expression in hepatocellular carcinoma on cell proliferation and tumorigenesis of NIH3T3 cells]. Beijing Da Xue Xue Bao 2003, 35(4):348-352.

36. Li L, Wei XH, Pan YP, Li HC, Yang H, He QH, Pang Y, Shan Y, Xiong FX, Shao GZ, Zhou RL: LAPTM4B: a novel cancer-associated gene motivates multidrug resistance through efflux and activating PI3K/AKT signaling. Oncogene 2010, 29(43):5785-5795.

37. Liu $X$, Xiong F, Wei $X$, Yang H, Zhou R: LAPTM4B-35, a novel tetratransmembrane protein and its PPRP motif play critical roles in proliferation and metastatic potential of hepatocellular carcinoma cells. Cancer Sci 2009, 100(12):2335-2340.

doi:10.1186/1471-2156-15-48

Cite this article as: Mo et al.: LAPTM4B polymorphism increases susceptibility to multiple cancers in Chinese populations: a meta-analysis. BMC Genetics 2014 15:48.

\section{Submit your next manuscript to BioMed Central and take full advantage of:}

- Convenient online submission

- Thorough peer review

- No space constraints or color figure charges

- Immediate publication on acceptance

- Inclusion in PubMed, CAS, Scopus and Google Scholar

- Research which is freely available for redistribution 